Classification

Physics Abstracts

$61.14-61.16-68.20$

\title{
Incoherence effects in reflection electron microscopy
}

\author{
Archie Howie $\left({ }^{1}\right)$, Mary Y. Lanzerotti $\left({ }^{1,2}\right)$ and Zhong Lin Wang $\left({ }^{3}\right)$ \\ ( $\left.{ }^{1}\right)$ Cavendish Laboratory, Madingley Road, Cambridge CB3 OHE, G.B. \\ (2) Department of Physics, Cornell University, Ithaca, New York 14583, U.S.A. \\ (3) Metals and Ceramics Division, Oak Ridge National Laboratory, P.O. Box 2008, Oak Ridge, \\ Tennessee 37831-6376, U.S.A.
}

(Received March 03, 1992; accepted May 06, 1992)

\begin{abstract}
The phase contrast imaging of surface steps in reflection electron microscopy is analysed. It is shown that the typical images are seriously affected by incoherence effects and that this arises, not from the strong inelastic scattering which is often present, but from the effective angular spread in the illumination which is frequently determined by the width of the Bragg reflection.
\end{abstract}

\section{Introduction.}

The magnetic prism system incorporated in the column of an electron microscope by Castaing and Henry [1] represented a pioneering advance in energy-selecting electron microscopy. Its potential for microanalysis was swiftly demonstrated [2] by the energy loss imaging of $\mathrm{Mg} \mathrm{Zn}$ precipitates in Al alloys using characteristic spectral features in the valence region. Although this approach to microanalysis is less readily made quantitative than currently more fashionable techniques in the STEM, the immediate high-quality output is valuable, particularly for zero-loss, energy-filtered images and has lead to the development of the omega filter system.

Of more relevance here are the beautiful observations which Castaing and his colleagues made of the effects of inelastic scattering on diffraction contrast, transmission images of crystals. Their energy loss images showed interference features such as Fresnel fringes [3], bend contours and thickness fringes [4] as well as stacking fault fringes [5] which were in many cases quite indistinguishable from the features of the zero-loss image. With a completeness which could not be matched by the alternative one-dimensional approach based on the Mollenstedt analyser [6, 7], these results from the Castaing-Henry system vindicated the theory [8] of coherence effects in energy loss images.

Diffraction contrast effects are generated by elastic scattering processes which are generally associated with atomic-scale spatial localisation. These processes act on electrons independently of whether they have also been inelastically scattered and hence their effects are visible in energyloss as well as zero-loss images. Particularly for low-loss, valence excitations, inelastic scattering 
processes are by comparison delocalised, corresponding to the relatively smaller typical scattering angles. Inelastic scattering can generate some additional contrast due to local variations in composition, but does not produce diffraction or interference contrast effects.

Although these ideas hold in more general cases [9] they can be elaborated more quantitatively in the case of crystals via the concepts of Bloch waves, dispersion surfaces and interband or intraband scattering processes $[8,10,11]$. Diffraction contrast can be well preserved, even in thick crystals where multiple inelastic scattering effects predominate. The gradual loss of contrast arises from the finite spread of inelastic scattering angles associated with the spatial localisation of the process $[11,12]$. This effect can be regarded as a form of increased spatial incoherence and becomes much more noticeable when images are formed at substantial defocus values $[7,13]$.

In the past two decades, interest in these topics has waned as new developments in transmission microscopy moved first to weak-beam techniques and then to high resolution imaging involving considerably thinner crystals where the effects of inelastic scattering no longer dominate attention. Nevertheless, the possibly significant contribution from high-angle inelastic scattering to weak beam images has been noted [14]. The presence of lattice structure contrast in high resolution loss images was demonstrated in the STEM [15] and also occurs in the TEM [16] but is complicated there by the dependence of defocus value on energy loss.

Reflection electron microscopy (REM) is another imaging mode which has been increasingly used in recent years [17]. Inelastic scattering effects can dominate the scattering at very small angles of grazing incidence and, even at the somewhat larger angles of incidence of over $30 \mathrm{mrad}$ typically used in practice, its influence can be far from negligible. Here we re-examine the origins of surface step contrast and investigate the effects of inelastic scattering and other sources of spatial incoherence on the REM images and reflection electron holograms of surface steps.

\section{Reflection electron images of surface steps.}

Imaging of surface steps, extending down to those of only monatomic height is now a widely used procedure [17] in reflection electron microscopy (REM). Since the step contrast reverses on either side of exact focus, where it vanishes or nearly vanishes, step visibility has from the outset been ascribed [18] to a phase contrast effect. Figure 1 is a typical example showing steps on the (111) surface of a single crystal of Pt imaged in the 444 reflection with $100 \mathrm{keV}$ electrons. Figures 1a, $1 \mathrm{~b}$ and $1 \mathrm{c}$ are taken in conditions of overfocus, near focus and underfocus respectively. However since the usual large foreshortening effect is present, a substantial variation in defocus (in the direction of underfocus) occurs from top to bottom of each individual image. The characteristically simple up-down or down-up image intensity profile of the steps is clearly visible, reversing on either side of exact focus.

It would be very useful to be able to interpret these surface step images more quantitatively to allow for instance the direct measurement of step heights. A more quantitative theory would also permit more conclusive identification of relaxation effects or surface reconstructions at surface steps. It has been suggested [19] for example that apparent changes of background contrast on either side of a step can be caused if the outermost layer or layers of atoms have an anomalous stacking arrangement. The dark oval regions visible in figure 1 could be an instance of such a phenomenon. An obvious test in the case of the (111) Pt surface is to check whether the contrast anomaly visible in images such as figure 1, taken near the [110] azimuth, disappears when imaged near the [112] azimuth. Though feasible, this difficult experiment has still not been done. In the case of very small ovals it is obviously not easy to distinguish a genuine anomaly in background contrast from the overlapping contrast of the steps bounding the oval. The first stage of any more quantitative theory has to be the evaluation of the phase shift across a step. 

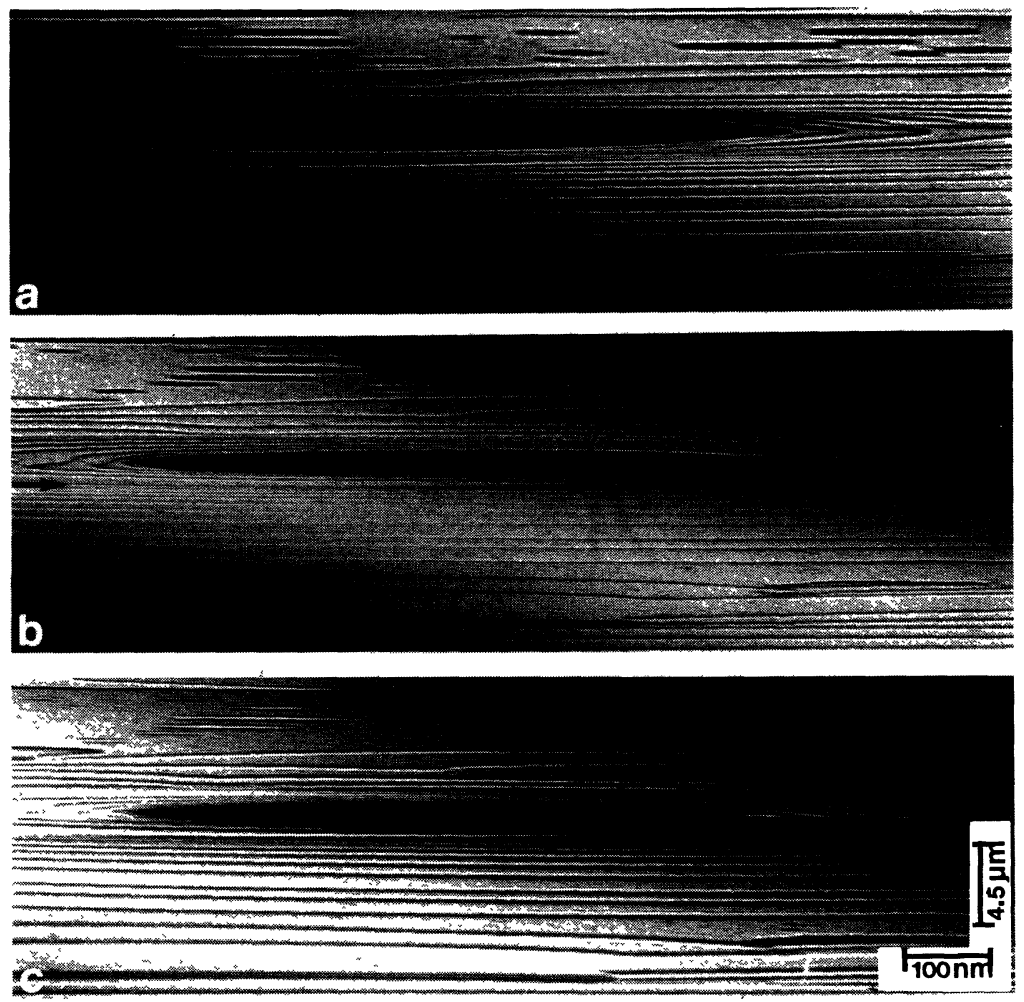

Fig. 1. - REM images of surface steps on the (111) surface of Pt, imaged in the 444 reflection near the [110] azimuth. Images (a) and (c) are taken in underfocus and overfocus conditions respectively. The exact focus condition occurs near the centre of figure $1 \mathrm{c}$ where the step contrast is very weak. The dark ovals referred to in the text here appear to lie in valleys but are often observed as islands at the top of hills.

\section{Phase shift at a step.}

In theory, the phase shift $\Delta \Phi$ across an unrelaxed step of height $H$ is given [20] by an expression readily deduced from figure 2 .

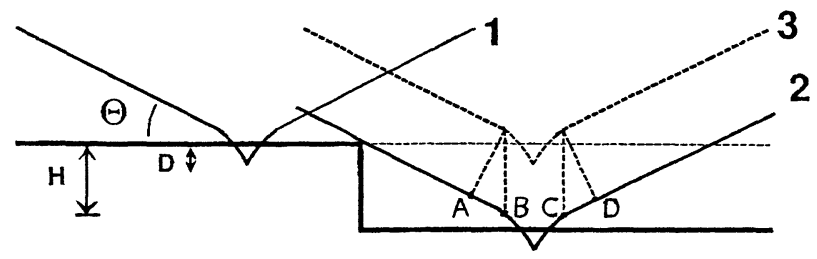

Fig. 2. Electron trajectories in REM including external deflection, refraction and finite penetration effects. The path difference between trajectories (1) and (2) is the same as that between (3) and (2) and can be seen to be $\mathrm{AB}+\mathrm{CD}=2 H \sin \theta$. 


$$
\Delta \Phi=\frac{4 \pi H \sin \theta}{\lambda}=2 \pi \frac{H}{d}\left(n^{2}-8 m \frac{d^{2}}{h^{2}}\left|V_{0}\right|\right)^{1 / 2}
$$

where $\theta$ is the angle of incidence and $\lambda$ the wavelength, both measured well outside the crystal which is assumed to be oriented to excite the $n^{\text {th }}$ order Bragg reflection from planes of spacing $d$ parallel to the crystal surface. $V_{0}$ is the inner potential energy, $h$ is Planck's constant and $m$ is the relativistically corrected electron mass. Since $n$ can often take values of 5 or more in typical REM experiments, $\Delta \Phi$ can be very large, even for monatomic steps $(H=d)$. An expression for $\Delta \Phi$ which is identical to equation (1) in the non-relativistic limit has also been quoted independently by Osakabe et al. [21]. Lehmpfuhl and Uchida [22] have derived, by a rather more complicated argument, yet another expression for $\Delta \Phi$ and given (in our notation) in terms of the energy $E$,

$$
\Delta \Phi=2 \pi d\left|V_{0}\right| /(\lambda E \sin \theta)
$$

Modulo $2 \pi$ however, expressions (1) and (2) are in practice quite similar at large values of $n$, as can be verified by expanding the square root in expression (1) and ignoring relativistic effects. To this kind of accuracy, these various expressions for $\Delta \Phi$ have indeed been experimentally confirmed by recent observations of fringe shifts at steps in reflection electron holograms [21,23].

The relevance of either equation (1) or (2) to the usual REM image of steps is much less clear however. There is not so far evidence for instance that typical images show the dependence on $n$ which equation (1) would suggest (see for example [18]). More crucially, the steps do not in general display the complex Fresnel profile with multiple subsidiary fringes which one would associate with any coherent out-of-focus image of an abrupt phase step of such magnitude. Image computations for such phase objects [24] do indeed exhibit a much more complex fringe structure than the basic up-down or down-up profile observed at surface steps in REM images. On the other hand, the simple ray diagram approach, indicated in figure 3 with refraction effects at the step edge, is able to explain qualitatively all of the observed effects, including the sign of the contrast as a function of the defocus condition and the up or down sense of the step relative to the beam direction. This argument is rather similar to the one originally given by Osakabe et al. [18] although, since they ignored the refraction effect in equation (1), they were driven to assume that the existence of any phase shift modulo $2 \pi$ across the step essentially arises from relaxation near the step. The success of this simple ray model of figure 3 suggests that long range phase shifts on either side of a step are nullified by some incoherence effect. The images apparently depend on the more local behaviour of the phase in the immediate vicinity of the step as represented by the phase gradient as a function of distance across the step (see Fig. 4). Typical REM images thus exhibit a partial degree of phase coherence which is quite remarkable. We need to identify the incoherence effect which destroys, but only partially destroys, the phase coherence.

\section{Temporal and spatial incoherence in REM images.}

The presence of strong inelastic scattering effects in REM images due to the excitation of surface, and to some extent bulk, plasmons is by now very well established [17, 20, 25, 26]. At typical angles of incidence $\theta \cong 30 \mathrm{mrad}$ the probability of valence excitation can be as great as 0.3 . Since the loss probabilities vary inversely with $\theta$, the probability of reflection without loss can become very small at small values of $\theta$ when multiple losses occur. Despire the strength of inelastic scattering, its effect on step contrast seems to be extremely small since energy selected reflection images taken with zero loss and surface plasmon loss in the STEM show effectively identical contrast [25]. 


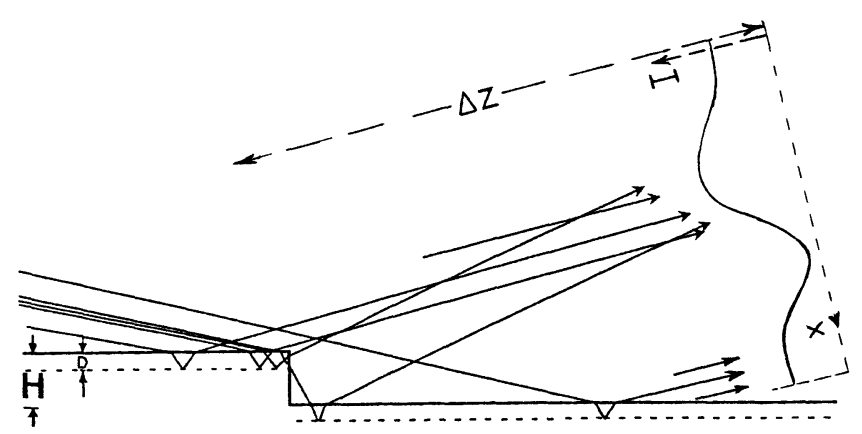

Fig. 3. - Ray paths near a step showing anomalous refraction effects leading to the typical up-down image profile in the overfocused image.

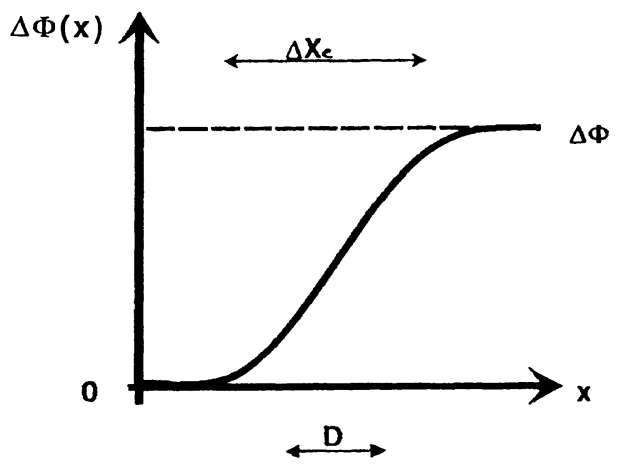

Fig. 4. - Schematic phase profile at a step as a function of distance in the projected image. The asymptotic phase shift is given by equation (1) but is built up gradually over a distance related to the penetration depth $D$ shown in figure 3.

An example taken from Bleloch et al. [26] is given in figure 5. This preservation of contrast after inelastic scattering is rather reminiscent of the well known situation in transmission electron microscopy (TEM). Apart from any TEM chromatic aberration effect, the images are quite insensitive to temporal incoherence because at any given energy loss, the same elastically scattered components are present to interfere with one another. In the STEM, the chromatic aberration effect is of course absent.

The small spread of inelastic scattering angles generates some spatial incoherence which becomes noticeable in TEM images [11], particularly at large defocus values [12, 13]. REM energy loss images are usually dominated by surface plasmon excitation, where the range of scattering angles is even smaller than for bulk plasmon excitation. Defocused, energy-loss REM images of steps do not therefore show significantly greater spatial incoherence than the zero-loss images. We may therefore conclude that the observed incoherence in most REM images arises not from the spread in energy or of inelastic scattering angles but must be due to the spatial incoherence of the illumination. 

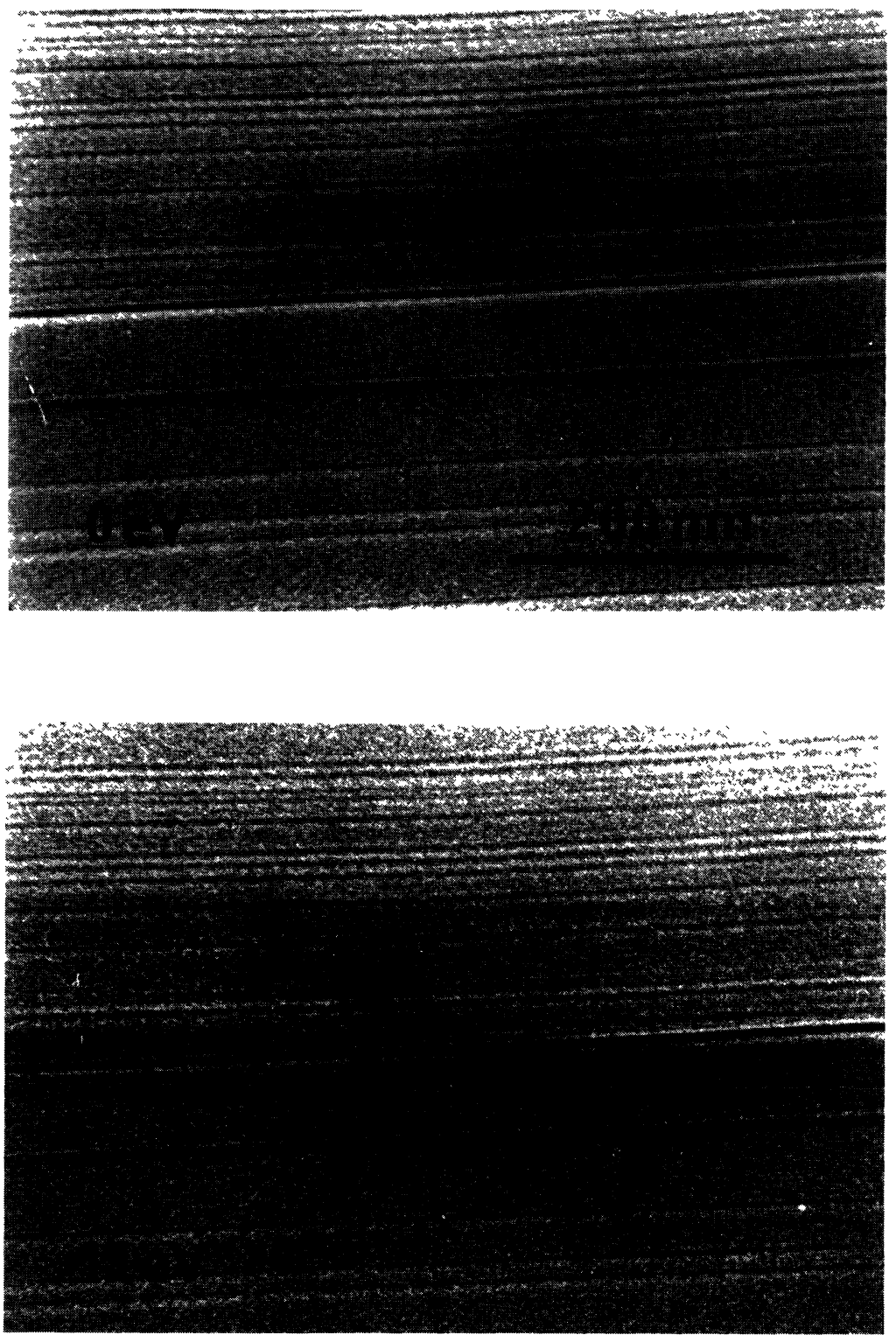

b)

Fig. 5. - REM images of the same area of a (110) GaAs surface at the 880 diffraction condition taken [26] in the zero loss (a) and $11 \mathrm{eV}$ surface plasmon loss (b). The two images are extremely similar apart for one or two fine details visible in (b) rather than in (a). Courtesy Plenum Press.

\section{Spatial incoherence in REM illumination.}

Bleloch et al. [20] suggested that the effective angular range of the illumination in REM might be limited to a range $\Delta \theta_{\mathrm{B}}$ by the width of the Bragg reflection and that, because of the finite penetration depth $D$, the phase jump at a step would take place over a finite distance. Figure 3 
suggests in fact that the phase jump at a small step should occur, as shown in figure 4 , over a distance $\Delta X=2 D$ in the projected image corresponding to a distance $\Delta X / \theta$ measured along the crystal surface across the step. However $\Delta \theta_{\mathrm{B}}$ and $D$ are themselves related, at least in kinematical theory, by the expression

$$
\theta_{\mathrm{B}} / \Delta \theta_{\mathrm{B}}=r=D / d
$$

where $r$ is the number of planes sampled. Typical values of $\theta_{\mathrm{B}} / \Delta \theta_{\mathrm{B}}=10[18]$ and of $D=2.5 \mathrm{~nm}$ [20] seem quite consistent with this expression. The corresponding spatial coherence distance can then be seen to be

$$
\Delta X_{\mathrm{c}}=\lambda / \Delta \theta_{\mathrm{B}}=\lambda D / d \theta_{\mathrm{B}}=2 D=\Delta X
$$

This expression shows that, unless the illumination has an angular spread $\Delta \theta<\Delta \theta_{\mathrm{B}}$, the angular filtering effect of the Bragg reflection will automatically determine a coherence distance which is rather similar to the distance of about $5 \mathrm{~nm}$ over which the phase variation across a step occurs. The occurence of phase contrast, but only limited phase contrast, in typical REM images of steps can thus be explained. One could expect that with more parallel illumination $(\Delta \theta<1 \mathrm{mrad})$ or at least more coherent illumination, multiple fringes would be observed at steps. Such images have indeed recently been obtained [27] using a field emission system. A further example of coherent step images taken with this system is shown in figure 6. From the width of these step images, it can be seen that the coherence distance $\Delta X_{\mathrm{c}}$ is about $100 \mathrm{~nm}$. The corresponding distance measured along the surface $\Delta X_{\mathrm{c}} / \theta$ is of course much greater.

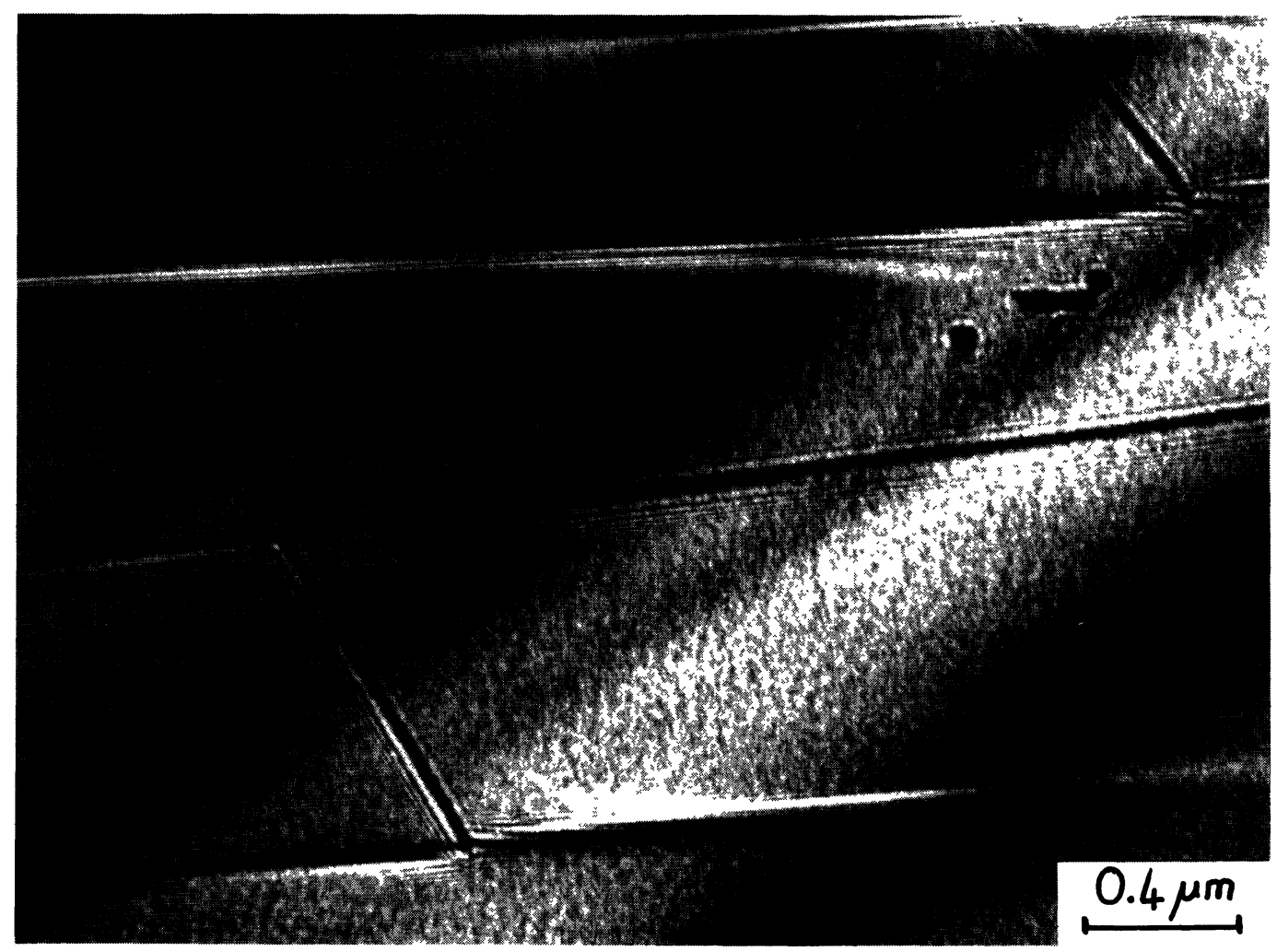

Fig. 6. - REM images of steps on a cleaved (011) surface of $\alpha-\mathrm{Al}_{2} \mathrm{O}_{3}$ taken in the $066 \overline{1} \overline{2}$ reflection with FEG illumination in a Philips 400 at $100 \mathrm{keV}$. 


\section{Discussion.}

For many purposes it may be preferable to continue to work with the familiar partially coherent REM images. The sense of each step is readily apparent and there is less problem with overlapping effects at images from neighbouring steps. There may also be greater image intensity available. The more coherent images available with field emission sources would appear to offer some advantages however. It should be possible for instance to determine the phase shift at a given step quite precisely and thus to measure the step height and detect any relaxation at the step. The influence of the order of the Bragg reflection used and of relativistic effects, as given by equation (1) could be analysed in detail. By quantitative analysis of the images as a function of defocus, a complete profile of the variation of the reflected amplitude and phase across the step could be determined.

For simplicity, we have considered the case of steps running normal to the electron beam. It is clear from figure 6 and other images however that rather similar arguments about partial coherence must also apply to conventional REM images of steps running more nearly parallel to the electron beam. Since REM images are frequently taken under resonance conditions where the azimuthal precision $\Delta \phi$ is comparable to the width of the Bragg reflection $\Delta \theta_{\mathrm{B}}$, we would expect a coherence distance $\Delta Y_{c}$, rather similar to $\Delta X_{c}$ in ordinary REM images. In coherent REM images $\Delta X_{\mathrm{c}}$ and $\Delta Y_{\mathrm{c}}$ would also be expected to be similar, since the angular spread of the illumination will usually be the same in the $X$ and $Y$ directions.

The reflection electron holograms which are now becoming available of course offer a unique test of the phase shift and phase coherence effects in REM. The gradual shift of the holographic fringes which is observed [21,23] over a distance of about $60 \mathrm{~nm}$ (measured along the crystal surface) seems to be consistent with the phase profile of figure 4 . So far however, the fringe displacements appear to demonstrate only the phase shift modulo $2 \pi$, possibly because of inadequate spatial resolution. Useful information about the extent of spatial coherence in the hologram could perhaps be obtained from REM images of the same step taken with the same angular spread of illumination as is used in the hologram. The question of temporal coherence in reflection electron holograms of steps is also an interesting one. It is not clear whether inelastically scattered electrons (which become very significant at smaller angles of incidence but are also present in the reference wave) can contribute to holographic fringes. For this to occur, a very high degree of spatial coherence in surface plasmon excitation would be required.

\section{Acknowledgements.}

We are grateful to the SERC for research support and to Plenum Press for permission to reproduce figure 5. ML thanks the U.S. Churchill Foundation for a studentship. ZLW was sponsored by the Division of Materials Sciences, US Dept. of Energy under contract DE-ACO5-84OR21400 with Martin Marietta Energy Systems inc.

\section{References}

[1] Castaing R. and Henry L., C.R. Hebd. Seanc. Acad. Sci. (Paris) 255 (1962) 76.

[2] Castaing R. and el Hili A., C.R. Hebd. Seanc. Acad. Sci. (Paris) 261 (1965) 3999.

[3] CASTAING R. and Henry L., C.R. Hebd. Seanc. Acad. Sci. (Paris) 259 (21964) 1702.

[4] Castaing R., el Hili A. and Henry L., C.R. Hebd. Seanc. Acad. Sci. (Paris) 262 (1966) 169. 
[5] Castaing R., Z. Angew. Phys. 27 (1969) 171.

[6] MOLLENSTEDT G., Optik 5 (1949) 499.

[7] Metherell A.J.F., Adv. Opt. El Micr., V.E. Cosslett and R. Barer Eds. (Academic Press) 4 (1971) 263.

[8] Howe A., Proc. Roy. Soc. A271 (1963) 268.

[9] HOWIE A., J. Microsc. 117 (1979) 11.

[10] HUMPHREYS C.J. and WHELAN M.J., Philos Mag. 20 (1969) 165.

[11] CUNDY S.L., HowIE A. and VALDRE U., Philos. Mag. 20 (1969) 147.

[12] METHERELL A.J.F., Philos. Mag. 16 (1967) 763.

[13] SPALDING D.R., PhD Thesis (Univ. of Cambridge) 1969.

[14] HowIE A., Proc. 39th EMSA Meeting (Clactors-Baton Roughe, 1981) p. 186.

[15] Craven A.J. and CollieX C., J. Microsc. Spectrosc. Electr. 1 (1977) 511.

[16] AJIKA N., HaShimoto H., YamaguCHi K. and Endo H., Jpn J. Appl. Phys. 24 (1985) L41.

[17] YAGI Y., J. Appl. Coyst. 20 (1987) 147.

[18] OSAKabe N., TANishiro Y., YaGi K. and Honjo G., Surf. Sci. 102 (1981) 424.

[19] TUNG HSU and COWLEY J.M., Structure of Surfaces (Springer-Berlin, 1985) p.55.

[20] BLELOCH A.L., HOWIE A., MILNE R.H. and WAllS M.G., Ultramicroscopy 29 (1989) 175.

[21] OSAKABE N., MATSUDA T., ENDO J. and TONOMURA A., Jpn J. Appl Phys. 27 (1988) L172.

[22] LEMPFUHL G. and UCHIDA Y., Surf. Sci. 188 (1987) 364.

[23] BANZHOF H., HeRrmann K.H. and Lichte H., EUREM 88 Conf. Proc., Inst. of Physics - Bristol (1988) p.263.

[24] COWLEY J.M. and PENG L.M., Ultramicroscopy 16 (1985) 59.

[25] HowIE A. and MILNE R.H., Ultramicroscopy 18 (1985) 427.

[26] BlelOCH A.L., HOWIE A., MilNE R.H. and WALLS M.G., Reflection High Energy Electron Diffraction and Refraction Imaging of Surfaces, P.K. Larsen and P.J. Dobson Eds. (Plenum Press-New York, 1988) pp. 77-89.

[27] WaNG Z.L. and Bentley J., Proc. 49th EMSA meeting (San Francisco Press, 1991) p.660. 